\title{
Purtscher-like Retinopathy Associated with Systemic Lupus Erythematosus
}

\section{Paradee Kunavisarut, Kessara Pathanapitoon \& Aniki Rothova}

To cite this article: Paradee Kunavisarut, Kessara Pathanapitoon \& Aniki Rothova (2016)

Purtscher-like Retinopathy Associated with Systemic Lupus Erythematosus, Ocular Immunology and Inflammation, 24:1, 60-68, DOI: 10.3109/09273948.2014.932816

To link to this article: http://dx.doi.org/10.3109/09273948.2014.932816

曲 Published online: 14 Jul 2014.

Submit your article to this journal $\pi$

WII Article views: 203

Q View related articles $₫$

View Crossmark data $\asymp$

4 Citing articles: 1 View citing articles 준 


\title{
Purtscher-like Retinopathy Associated with Systemic Lupus Erythematosus
}

\author{
Paradee Kunavisarut, $\mathrm{MD}^{1}$, Kessara Pathanapitoon, $\mathrm{MD}, \mathrm{PhD}^{1}$, and \\ Aniki Rothova, $\mathrm{MD}, \mathrm{PhD}^{2}$ \\ ${ }^{1}$ Department of Ophthalmology, Faculty of Medicine, Chiang Mai University, Chiang Mai, Thailand and \\ ${ }^{2}$ Department of Ophthalmology, Erasmus Medical Center, Rotterdam, The Netherlands
}

\begin{abstract}
Purpose: To report on clinical manifestations of Purtscher-like retinopathy (PLR) associated with systemic lupus erythematosus (SLE) and visual outcomes.

Methods: We performed a retrospective cohort study of 11 patients (21 affected eyes) with PLR in SLE.

Results: All patients were treated with systemic corticosteroids \pm immunosuppressive agents. Ocular therapy included intravitreal injections with bevacizumab in 18/21 eyes and posterior sub-Tenon injections with triamcinolone acetonide 13/21 eyes. Panretinal photocoagulation (PRP) was performed in 19/21 eyes and pars plana vitrectomy was required in 5/21 eyes. Visual improvement was found at follow-up of 3 and 6 months $(p=0.05)$. Poor visual outcome was associated with presence of neovascularizations at onset $(p=0.009)$, development of vitreous hemorrhage during PRP $(p=0.015)$, and active status of SLE after onset of PLR $(p=0.029)$. Conclusions: PLR might manifest as a devastating complication of SLE. We recommend treating any systemic activity of SLE and starting an early ocular treatment.
\end{abstract}

Keywords: Bevacuzimab, complication, purtscher-like retinopathy, systemic lupus erythematosus, treatment

Purtscher's retinopathy is a clinical syndrome characterized by ischemic changes of the posterior pole associated with multiple areas of whitening of inner retina (Purtscher flecken), optic disc swelling, cotton wool spots, and hemorrhages usually concentrated around the optic disc. Purtscher's retinopathy is a mostly bilateral disorder and was originally described after various types of trauma. Pathogenesis of this syndrome was attributed to embolic occlusions of the precapillary arterioles and/or impaired venous return from raised intracranial/intrathoracic pressure and secondary extravasation of fluid. ${ }^{1-3}$

Purtscher-like retinopathy (PLR) represents a clinical syndrome with manifestations similar to the original Purtscher's retinopathy but related to causes other than trauma. ${ }^{3}$ PLR was reported in various conditions such as acute pancreatitis, ${ }^{4-6}$ fat embolism, ${ }^{7}$ chronic renal failure, ${ }^{8}$ hemolytic uremic syndrome, ${ }^{9,10}$ after childbirth, ${ }^{11}$ weight lifting, ${ }^{12}$ battered child syndrome, ${ }^{13}$ and several autoimmune diseases. ${ }^{14,15}$ The visual prognosis of PLR in general is poor and the secondary development of neovascularizations and glaucoma might even destroy the eye.

Herein we report on PLR in 11 patients with systemic lupus erythematosus (SLE) and refer to its management and visual outcomes.

\section{MATERIALS AND METHODS}

In this series we included 11 consecutive patients with PLR associated with SLE from the ophthalmological department of Chiang Mai University Hospital in Thailand between September 2006 and September 2012. We performed a retrospective study of their clinical, laboratory, and imaging data. We registered clinical ocular manifestations, development of visual acuity (VA) over time, complications, and SLE activity,

Received 1 May 2014; revised 4 June 2014; accepted 5 June 2014; published online 14 July 2014

Correspondence: Paradee Kunavisarut, MD, Dept. of Ophthalmology, Faculty of Medicine, 110 Intawaroros Road, Chiang Mai 50200, Thailand. E-mail: pkunavis@mail.med.cmu.ac.th or pkunavisarut@hotmail.com

Color versions of one or more of the figures in the article can be found online at www.tandfonline.com/ioii. 
as well as systemic and ocular treatment regimens employed. Mean follow-up time was 19 months (median 10 months; SD 20.051; range 3-72 months).

The diagnosis of SLE was based on the American College of Rheumatology criteria in all cases. ${ }^{16}$ The SLE disease activity was determined using the MEXSLEDAI score. ${ }^{17}$ PLR was defined as ischemia of the posterior pole associated with multiple areas of whitening of inner retina with optic disc swelling, cotton wool spots, and hemorrhages in the absence of previous trauma.

All patients were treated with a combination of systemic (oral or intravenous corticosteroids, sometimes with addition of other immunosuppressive agents) and local treatment (posterior sub-Tenon corticosteroid and/or anti-vascular endothelial growth factor (anti-VEGF) intraocular injections, panretinal photocoagulation, and occasionally pars plana vitrectomy), depending on the clinical condition of the patients. First, we attempted to control the activity of systemic SLE and PLR with a combination of systemic and local treatment, then we applied antiVEGF injections in patients with neovascularizations, large areas of nonperfusion on fluorescein angiography (FA), and/or macular edema. Panretinal photocoagulation (PRP) was performed in all cases with neovascularizations and/or large area of nonperfusion on FA. Pars plana vitrectomy (PPV) was performed in cases resistant to the above indicated treatment or in patients with vitreous hemorrhage. During follow-up, all patients were regularly evaluated by gonioscopy for the presence of neovascularizations.

We defined the progression of PLR as the ongoing development of one or more of following characteristics: retinal neovascularization, vitreous hemorrhage, neovascular glaucoma, progression of retinal ischemic areas, and increase of macular edema. Stabilization of PLR was defined as a situation in which no progression occurred and hemorrhages and neovascularizationd diminished.

For all calculations with VA data, we converted Snellen VA to the logarithm of the minimum angle of resolution (logMAR). For easier understanding the $\log$ MAR results were converted back to Snellen VA and presented in this report. We included only right eyes of affected patients for all calculations to prevent the bias of similar manifestations in bilateral cases.

This study was approved by a suitably constituted ethics committee of the institution within which the work was undertaken and conforms to the provisions of the Declaration of Helsinki.

\section{RESULTS}

General characteristics of the patients are given in Table 1. All patients except one were females
TABLE 1. General features of 11 patients with Purtscher-like retinopathy associated with systemic lupus erythematosus.

\begin{tabular}{lc}
\hline Median age (years) & 29.0 (range 15-44) \\
Female gender & $10(91 \%)$ \\
Bilateral involvement & $10(91 \%)$ \\
Known with SLE before the & $5(45 \%)$ \\
$\quad$ onset of PLR & $7(64 \%)$ \\
Active systemic SLE at onset of PLR & $6(55 \%)$ \\
Systemic medication at onset of PLR & $1(9.5 \%)$ \\
Positive anticardiolipin antibodies & 56.0 range $(16-118)$ \\
Median ESR & 10.0 range (3-72) \\
\hline Median follow-up time (months) & \\
\hline
\end{tabular}

SLE, systemic lupus erythematosus; PLR, Purtscher-like retinopathy; ESR, erythrocyte sediment rate.

(10/11 patients; $91 \%)$ with a mean age of 31 years (median 29 years; SD 9.792; range 15-44 years). Before the onset of PLR, 5/11 (45\%) patients were already known to have SLE and the remaining 6/11 (55\%) were diagnosed with SLE after the onset of PLR. At the time of PLR onset, active SLE was observed in $7 / 11$ patients $(64 \%)$ and the remaining 4 were considered to be in the inactive stage. Anticardiolipin antibodies were positive only in 1 patient $(9.5 \%)$. None had additional risk factors for retinal ischemia, such as diabetes mellitus, hypertension, dyslipidemia, or nephropathy.

Bilateral ocular involvement was present in 10/11 (91\%) patients, resulting in 21 affected eyes. FA was performed in 9/11 patients (out of 2 missing cases, one had known allergy to fluorescein and the other had dense vitreous hemorrhages, which prevented view of the fundus). Macular ischemia and a nonperfusion area of more than 10 disc diameters were noted at onset in 16/18 (89\%) evaluable eyes (Figures 1, 2). Retinal neovascularization at initial presentation was demonstrated in $4 / 11(36 \%)$ patients (7/21;33\% affected eyes).

After the onset of PLR, all patients were treated with systemic steroids ( $1 \mathrm{mg} / \mathrm{kg} /$ day), and additional immunosuppressive treatment was initiated in 6 patients, resulting in 7 patients being on immunosuppressive therapy (Table 2). Severe systemic complications caused by either SLE or its treatment developed in 3 patients (sepsis in all, Table 2).

Ocular treatment included intravitreal injections with bevacizumab (Avastin $1.25 \mathrm{mg} / 0.05 \mathrm{~mL}$ ) and posterior sub-Tenon injections with triamcinolone acetonide (Kenakort $20 \mathrm{mg} / 0.5 \mathrm{~mL})(18 / 21 ; 86 \%$ and $13 / 21 ; 62 \%$ of affected eyes, respectively). Panretinal photocoagulation (PRP) was performed in 19/21 (91\%) of affected eyes. Pars plana vitrectomy (PPV) was performed in 4 patients; $5 / 21(24 \%)$ of affected eyes, which was $1 / 5$ eyes (case 7 ), had severe vitreous hemorrhage at the initial presentation.

With a combined systemic and ocular treatment, the progression of PLR was stopped in all patients. There were $2 / 11$ patients $(18 \% ; 4 / 21$ affected eyes, 

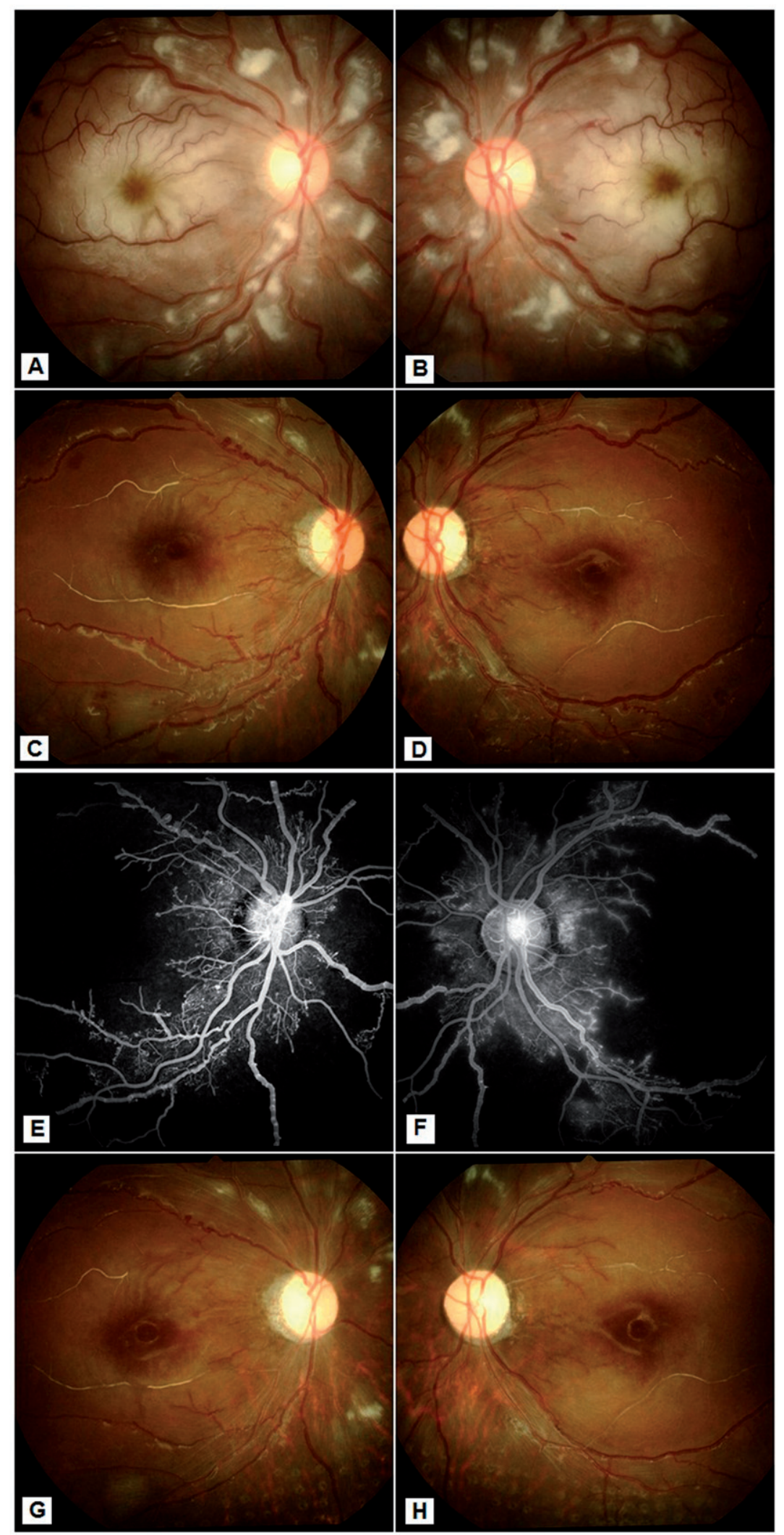

FIGURE 1. Ocular manifestations of Purtscher-like retinopathy associated with systemic lupus erythematosus (Table 2, case 2). (A, B) Fundus photographs of both eyes at presentation demonstrate extensive cotton wool spots and occlusions of multiple arterioles, including the macular area, which exhibits cherry red spots. (C-F) Fundus photographs and fluorescein angiography at 3 months follow-up, after treatment with oral prednisolone and cyclophosphamide, the cotton wool spots subsided but disc and retinal neovascularizations developed. Fundus fluorescein angiography shows extensive ischemia involving macula and neovascularizations. $(\mathrm{G}, \mathrm{H})$ Fundus photographs at 9 months follow-up. After panretinal laser photocoagulation as well as intraocular injections with bevacizumab, the neovascularizations regressed and the situation stabilized with bilateral pale optic discs. 


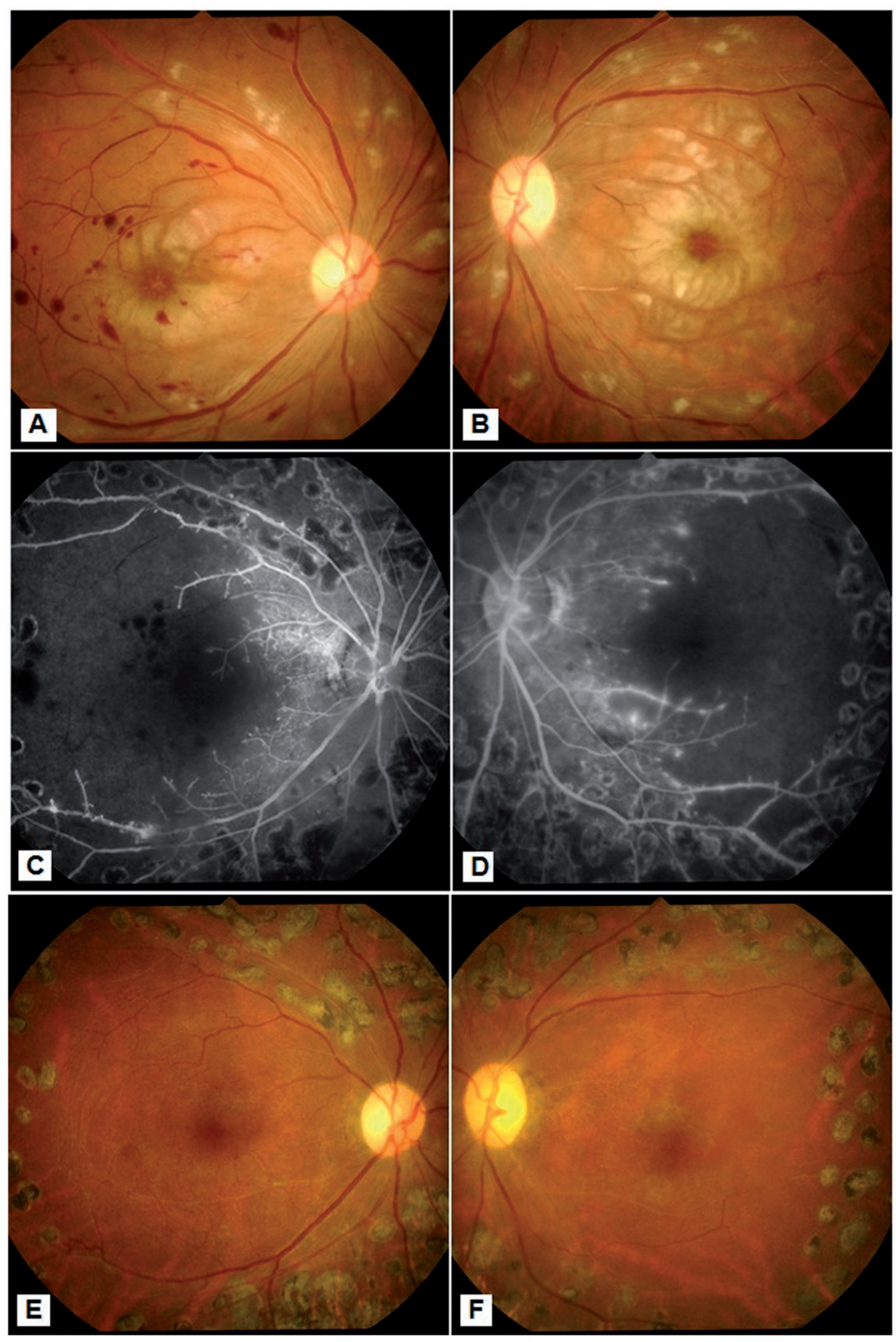

FIGURE 2. Ocular manifestations of Purtscher-like Retinopathy associated with systemic lupus erythematosus (Table 2, case 3). (A, B) Fundus photographs of both eyes at presentation demonstrate extensive cotton wool spots and occlusions of multiple arterioles, including macular area, which exhibits cherry red spots. (C, D) Fundus fluorescein angiography at 5 months follow-up, after panretinal laser photocoagulation as well as intraocular injections with bevacizumab, shows large areas of nonperfusion, vascular leakage, and macular ischemia without neovascularization. (E, F) Fundus photographs at 1 year follow-up, when retinal situation became quiescent. 


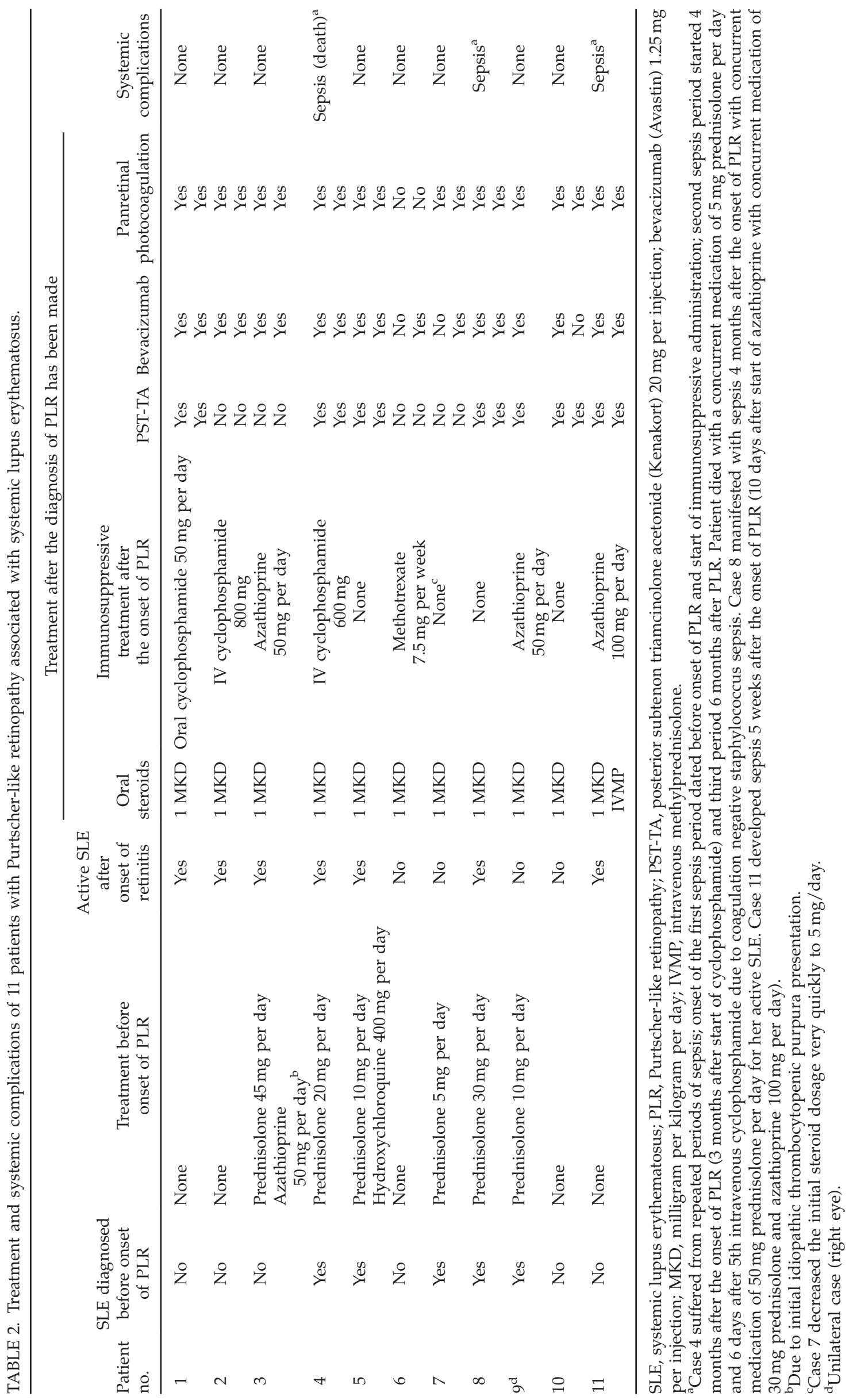


19\%) who stabilized within 3 months and 9 patients (82\%; 15/21 eyes; $71 \%$ ) reached a stabilized situation within 6 months. During PRP, vitreous hemorrhage occurred in 4/11 patients $(7 / 21$ eyes, of whom 4 required PPV and additional laser coagulation), but retinal detachments did not develop. Additional complications included ocular hypertension in 3/11 patients (4/21 eyes), which could be controlled with one or two topical anti-glaucoma medications, but none of the patients developed neovascular glaucoma.

At onset, visual acuity (VA) of less than 0.1 was found in the majority of patients $(8 / 11 ; 73 \% ; 14 / 21$; $67 \%$ eyes). At 6 months follow-up, VA of less 0.1 was noted in $5 / 9(56 \%)$ patients $(8 / 17 ; 47 \%$ eyes). After treatment, the most affected eyes $(14 / 21 ; 67 \%)$ had improved visual acuity while 4/21 (19\%) had stable VA and 3/21 (14\%) decreased. While we observed significant visual improvement at 3 and 6 months of follow-up ( $p=0.05$, Wilcoxon signed rank test), the number of eyes with VA of less than 0.1 at onset and at 6 months follow-up did not differ $(14 / 21 ; 67 \%$ versus $8 / 17,47 \%, p=0.324)$. VA of less than 0.1 at 6 months follow-up was associated with following characteristics: the presence of neovascularization at onset of PLR ( $p=0.009$ Fisher's exact test), the development of vitreous hemorrhage during PRP ( $p=0.015$ Fisher's exact test), and active status of SLE after the onset of PLR ( $p=0.029$ Fisher's exact test). No associations were observed between the visual outcomes and activity of SLE at onset of PLR, medications at onset, presence of anticardiolipin antibodies, treatment with immunosuppressive agents, area of nonperfusion, presence of macular ischemia, and initial VA.

Since our treatment induced stabilization in all eyes, oral prednisolone medication could be gradually decreased and/or tapered off in all patients. Six out of 11 patients could stop prednisolone medication entirely (mean 21 months; median 13 months; range 8-60 months) and the remainder could decrease their daily dosage. During the follow-up, 3 out of 11 patients developed sepsis at various intervals after the onset of PLR (Table 2) and 1 of these patients died. The septic periods developed at variable times after the onset of PLR in patients with different therapeutic regimens and, moreover, 1 patient suffered from septic phases even before the onset of PLR.

\section{DISCUSSION}

We report on severe PLR in 11 patients with SLE and point out that stabilization of PLR occurred in all cases with a combination of systemic and local treatment regimens. All but 1 patient had bilateral PLR and a majority of patients $(73 \%)$ had visual acuity at onset of less than 0.1. During follow-up, none of the patients developed retinal detachment or neovascular glaucoma, which might be caused by an aggressive treatment including the early local application of bevacizumab.

Ocular complications of SLE are generally associated with active systemic disease and the pathogenic mechanism of the SLE retinopathy has been attributed to the immune complex-mediated vasculopathy. ${ }^{18-20}$ Therefore, control of the systemic disease is a primary goal of the treatment. The aggressive immunosuppression was recommended, which seemed to improve the systemic disease and the status of retinopathy but visual outcome was generally unaffected due to persistent arteriolar attenuation and subsequent progression of retinal vascular occlusions. ${ }^{21-24}$

Ischemia and neovascularizations in PLR were regularly reported (in our series illustrated in Figure 1 of case 2) resulting in very poor visual outcomes. Miguel et al. and Sellami et al. compared the visual outcomes of PLR of diverse origins and concluded that SLE-associated PLR had worst visual acuity at presentation (1.7 $\log M A R)$ and did not improve with corticosteroid treatment. ${ }^{15,25}$ Previously, approximately $50 \%$ of SLE patients with PLR had visual acuity less than $6 / 20 .{ }^{26-29}$ Jabs et al. ${ }^{21}$ reported on this potentially visually devastating form of retinopathy in SLE in 11 patients (20 involved eyes) and noted the very poor visual outcomes despite the use of different regimens of immunosuppressive drugs, including oral and intravenous corticosteroids, azathioprine, and cyclophosphamide. In his series from the pre-anti VEGF era, approximately $50 \%$ of involved eyes had visual acuity less than 6/60, and $45 \%$ developed vitreous hemorrhage, which is closely similar to our study (47 and 38\%, respectively). In contrast to our series, the authors also observed retinal detachment in 3 eyes (15\%), and 1 additional eye $(5 \%)$ developed ischemia of anterior eye segment. The severity of PLR in our patients seems to be similar to that in previous reports, but might have been also influenced by a late presentation since one-third of the affected eyes had already retinal neovascularizations at first presentation to ophthalmologist. Despite this severe initial presentation, the retinal manifestations stabilized in all patients and lacked severe complications such as neovascular glaucoma, retinal detachment, and anterior segment ischemia.

The role of bevacizumab may explain this difference in outcome. Bevacizumab induces temporary decrease of VEGF levels and reduces retinal nonperfusion, which might cover the time interval until the PRP became effective. Campochiaro et al. ${ }^{30}$ found that high levels of VEGF result in worsening of retinal ischemia and progressive retinal nonperfusion, including the macular area. Therefore, anti-VEGF medications can reduce the total amount of retinal nonperfusion/macular edema and counteract the neovascular complications. ${ }^{30}$ Obviously, bevacizumab cannot restore the obliteration of the arteries involved 


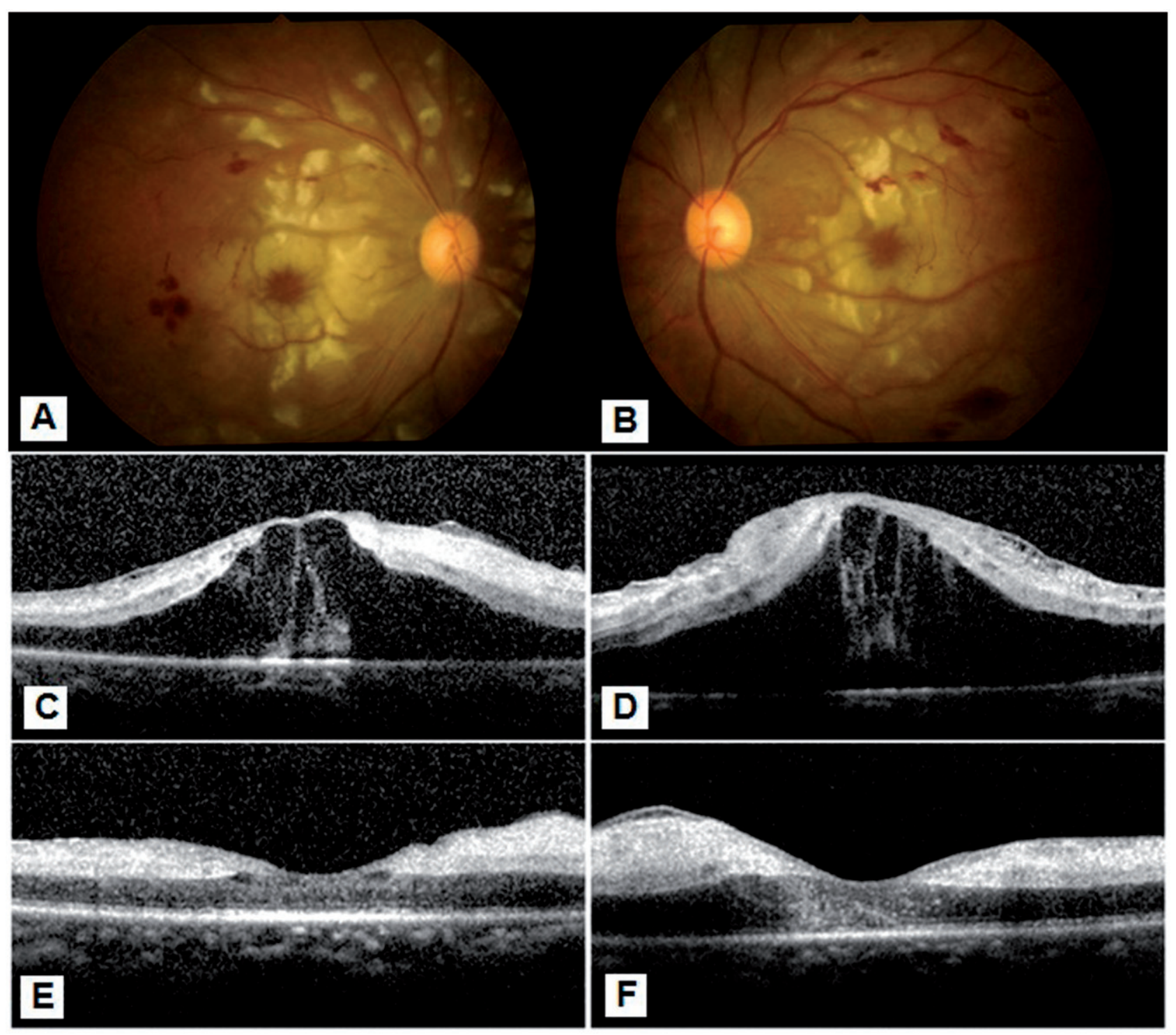

FIGURE 3. Ocular manifestations of Purtscher-like retinopathy associated with systemic lupus erythematosus (Table 2, case 11). (A-D) Fundus photographs and optical coherence tomography at presentation exhibit cotton wool spots, hemorhages together with bilateral macular edema. (E, F) Optical coherence tomography 3 weeks after intravitreal bevacizumab injection demonstrates the resolution of macular edema. During an additional 3 months follow-up, macular edema did not recur but visual acuity did not improve due to macular ischemia.

and the visual outcome remains dependent of macular ischemic changes (Figure 3, case 11).

Aggressive treatment of SLE has effectively reduced the mortality related to disease activity but also has led to the occurrence of complications that can be related to disease itself or to adverse effects of treatment, especially infections related to severe immunosuppression. Mortality rates in SLE cohorts with follow-up periods ranging from 8 to 14 years varied from 6.8 to $20.2 \% .^{31-34}$ The reported causes of death from SLE differ widely. While some studies reported that activity of SLE represented a major cause of death, ${ }^{35-37}$ others found that infections were more common. ${ }^{34,38-42}$ However, most patients who died from infections also had active SLE. Our 3 patients who developed sepsis received different therapeutic regimens and it was not possible to conclude whether sepsis developed due to severe active SLE or due to its treatment. Two patients had septic periods already before the start of immunosuppressive treatment and moreover developed septic stage during the decreasing medications a long time after the onset of PLR. One patient, however, developed sepsis 10 days after the initiation of azathioprine treatment given because of PLR. In a study of 349 Thai patients with SLE, the patients with infectionrelated and not infectious causes of death did not differ in the dosages of both prednisolone and/or cyclophosphamide. $^{34}$

The retrospective nature of the study and the absence of a control group constitute the drawbacks of our study. Based on our results it is difficult to prove 
that combination of systemic and aggressive ocular treatment including bevacizumab injection can stop the progression of PLR. However, case-control or randomization trials will probably not be feasible in the uncommon SLE complication. However, the stabilization of PLR and absence of neovascular glaucoma suggest that our treatment approach might be effective.

We conclude that PLR might manifest as a devastating ocular complication of SLE and recommend treating the systemic SLE activity in all affected patients and starting an early local treatment with anti-VEGF medications and PRP in order to retain as much useful vision as possible.

\section{ACKNOWLEDGEMENTS}

We thank Anjo Riemens, MD, for her help with statistics.

\section{DECLARATION OF INTEREST}

The authors report no conflicts of interest. The authors alone are responsible for the content and writing of the paper.

\section{REFERENCES}

1. Behrens-Baumann W, Scheurer G, Schroer H. Pathogenesis of Purtscher's retinopathy: an experimental study. Graefes Arch Clin Exp Ophthalmol. 1992;230:286-291.

2. Lai JC, Johnson MW, Martonyi CL, Till GO. Complement induced retinal arteriolar occlusions in the cat. Retina. 1997; 17:239-246.

3. Agrawal A \& McKibbin MA. Purtscher's and Purtscherlike retinopathies: a review. Surv Ophthalmol. 2006;51: 129-136.

4. Bui SK, O'Brien JM, Cunningham ET. Purtscher's retinopathy following drug-induced pancreatitis in an HIVpositive patient. Retina. 2001;21:542-545.

5. Campo SM, Gasparri V, Catarinelli G, Sepe M. Acute pancreatitis with Purtscher's retinopathy: case report and review of the literature. Dig Liver Dis. 2000;32:729-732.

6. Jacob HS, Goldstein IM, Shapiro I, et al. Sudden blindness in acute pancreatitis: possible role of complement-induced retinal leukoembolization. Arch Intern Med. 1981;141: 134-136.

7. Lemagne JM, Michiels X, Van Causenbroeck S, Snyers B. Purtscher-like retinopathy after retrobulbar anesthesia. Ophthalmology. 1990;97:859-861.

8. Stoumbos VD, Klein ML, Goodman S. Purtscher-like retinopathy in chronic renal failure. Ophthalmology. 1992; 99:1833-1839.

9. Lauer AK, Klein ML, Kovarik WD, Palmer EA. Hemolytic uremic syndrome associated with Purtscher-like retinopathy. Arch Ophthalmol. 1998;116:1119-1120.

10. Patel MR, Bains AK, O'Hara JP, et al. Purtscher's retinopathy as the initial sign of thrombotic thrombocytopenic purpura/hemolytic uremic syndrome. Arch Ophthalmol. 2001;119:1388-1389.
11. Blodi BA, Johnson MW, Gass JD, et al. Purtscher's-like retinopathy after childbirth. Ophthalmology. 1990;97: 1654-1659.

12. Kocak N, Kaynak S, Kaynak T, et al. Unilateral Purtscherlike retinopathy after weight-lifting. Eur J Ophthalmol. 2003; 13:395-397.

13. Tomasi LG, Rosman NP. Purtscher's retinopathy in the battered child syndrome. Am J Dis Child. 1975;129: 1335-1337.

14. Myers JP, Di Bisceglie AM, Mann ES. Cryoglobulinemia associated with Purtscher-like retinopathy. Am J Ophthalmol. 2001;131:802-804.

15. Sellami D, Ben Zina Z, Jelliti B, et al. Purtscher-like retinopathy in systemic lupus erythematosus; two cases. J Fr Ophtalmol. 2002;25:52-55.

16. Tan EM, Cohen AS, Fries JF, et al. The 1982 revised criteria for the classification of systemic lupus erythematosus. Arthritis Rheum. 1982;25:1271-1277.

17. Guzman J, Cardiel MH, Arce-Salinas A, et al. Measurement of disease activity in systemic lupus erythematosus: prospective validation of 3 clinical indices. J Rheumatol. 1992;19:1551-1558.

18. Peponis V, Kyttaris VC, Tyradellis C, et al. Ocular manifestations of systemic lupus erythematosus: a clinical review. Lupus. 2006;15:3-12.

19. Read RW. Clinical mini-review: systemic lupus erythematosus and the eye. Ocul Immunol Inflamm. 2004;12:87-99.

20. Nag TC, Wadhwa S. Vascular changes of the retina and choroids in SLE: pathology and pathogenesis. Curr Neurovasc Res. 2006;3:159-168.

21. Jabs DA, Fine SL, Hochberg MC, et al. Severe retinal vasoocclusive disease in systemic lupus erythematosus. Arch Ophthalmol. 1986;104:558-563.

22. Kayazawa F, Honda A. Severe retinal vascular lesions in systemic lupus erythematosus. Ann Ophthalmol. 1981;13: 1291-1294.

23. Lantham JG, Barrie T, Kohner EM, Hughes GRV. SLE retinopathy: evaluation by fluorescein angiography. Ann Rheum Dis. 1982;41:473-478.

24. Read RW, Chong LP, Rao NA. Occlusive retinal vasculitis associated with systemic lupus erythematosus. Arch Ophthalmol. 2000;118:588-589.

25. Miguel A, Henriques F, Azevedo LF, et al. Systematic review of Purtscher's and Purtscher-like retinopathies. Eye. 2013;27:1-13.

26. Au A, O'Day J. Review of severe vaso-occlusive retinopathy in systemic lupus erythematosus and the antiphospholipid syndrome: associations, visual outcomes, complications and treatment. Clin Experiment Ophthalmol. 2004;32:87-100.

27. Montehermoso A, Cervera R, Font J, et al. Association of antiphospholipid antibodies with retinal vascular disease in systemic lupus erythematosus. Semin Arthritis Rheum. 1999:28:326-332.

28. Asherson RA, Merry P, Acheson JF, et al. Antiphospholipid antibodies: a risk factor for occlusive vascular disease in systemic lupus erythematosus and the 'primary' antiphospholipid syndrome. Ann Rheum Dis. 1989; 48:358-361.

29. Fitzpatrick EP, Chesen N, Rahn E. The lupus anticoagulant and retinal vaso-occlusive disease. Ann Ophthalmol. 1990; 22:148-152.

30. Campochiaro PA, Bhisitkul RB, Shapiro H, Rubio RG. Vascular endothelial growth factor promotes progressive retinal nonperfusion in patients with retinal vein occlusion. Ophthalmology. 2013;120:795-802.

31. Cervera R, Khamashta MA, Font J, et al. Morbidity and mortality in systemic lupus erythematosus during a 10-year period: a comparison of early and late manifestation in a cohort of 1000 patients. Medicine. 2003;82:299-308. 
32. Moss KE, Ioannou Y, Sultan SM, et al. Outcome of a cohort of 300 patients with systemic lupus erythematosus attending a dedicated clinic for over two decades. Ann Rheum Dis. 2002;61:409-413.

33. Yeap SS, Chow SK, Manivasagar M, et al. Mortality pattern in Malaysian systemic lupus erythematosus. Med J Malaysia. 2001;56:308-312.

34. Kasitanon N, Louthrenoo W, Sukitawut W, Vichainun R. Causes of death and prognostic factors in Thai patients with systemic lupus erythematosus. Asian Pacific J Allergy Immunol. 2002;20:85-91.

35. Ward MM, Pyun E, Studenski S. Causes of death in systemic lupus erythematosus: long-term follow up of an inception cohort. Arthritis Rheum. 1995;38:1492-1499.

36. Koh ET, Seow A, Leong KH, Chng HH. SLE mortality in an oriental population. Lupus. 1997;6:27-31.

37. Pistiner M, Wallace DJ, Nessim S, et al. Lupus erythematosus in the 1980s: a survey of 570 patients. Semin Arthritis Rheum. 1991;21:55-64.
38. Doria A, Iaccarino L, Ghirardello A, et al. Long-term prognosis and causes of death in systemic lupus erythematosus. Am J Med. 2006;119:700-706.

39. Kim WU, Min JK, Lee SH, et al. Causes of death in Korean patients with systemic lupus erythematosus: a single center retrospective study. Clin Exp Rheumatol. 1999;17: 539-545.

40. Ginzler EM, Diamond HS, Weiner M, et al. A multicenter study of outcome in systemic lupus erythematosus, I: entry variables as predictors of prognosis. Arthritis Rheum. 1982; 25:601-611.

41. Rosner S, Ginzler EM, Diamond HS, et al. A multicenter study of outcome in systemic lupus erythematosus, II: causes of death. Arthritis Rheum. 1982; 25:612-617.

42. Mok CC, Mak A, Chu WP, et al. Long-term survival of southern Chinese patients with systemic lupus erythematosus: a prospective study of all age groups. Medicine. 2005; 84:218-224. 